\title{
Evolução das publicações em saúde do idoso na Revista Ciência \& Saúde Coletiva
}

\author{
Evolution of publications on health of the older adults \\ in the Journal Ciência \& Saúde Coletiva
}

Josélia Oliveira Araújo Firmo (https://orcid.org/0000-0001-5330-476X) ${ }^{1}$

Sérgio Viana Peixoto (https://orcid.org/0000-0001-9431-2280) 1,2

Gislaine Alves de Souza (https://orcid.org/0000-0002-4556-2416) ${ }^{3}$

Antônio Ignácio de Loyola Filho (https://orcid.org/0000-0002-7317-3477) 1,2
${ }^{1}$ Núcleo de Estudos em Saúde Pública e Envelhecimento, Instituto René Rachou, Fiocruz Minas. Av. Augusto de Lima 1715, Barro Preto. 30190-002 Belo Horizonte MG Brasil. joselia.firmo@fiocruz.br ${ }^{2}$ Escola de Enfermagem, Universidade Federal de Minas Gerais. Belo Horizonte MG Brasil. ${ }^{3}$ Programa de PósGraduação em Saúde Coletiva, Instituto René Rachou, Fiocruz Minas. Belo Horizonte MG Brasil.
Abstract The present study analyzed the profile of publications related to the health of the older adults in the Journal Ciência \& Saúde Coletiva, in terms of quantitative evolution, methodological approach, thematic and institutions involved. The selection of articles considered the title, abstract and descriptors and covered all the content published between 1996 and 2019. The articles included were classified according to the year of publication, type, theme, methodological approach and institutional link of the first author. Data analysis was based on absolute and relative frequencies, in addition to summary (mean) and variability (standard deviation) measures. The results showed a consistent increase in publications on the health of the older adults in the Journal Ciência \& Saúde Coletiva, with a predominance of the quantitative approach, mainly focused on investigating the topic related to health conditions and the use of health services and supplies. In the majority, the main author was linked to a public teaching/research institution, located in the Southeast region. The results indicate that the journal contributed to give greater visibility to the health of the older adults, but that the publications on this theme reproduce the inequality observed in the national scientific production.

Key words Health of the Older Adults, Aging, Scientific publications
Resumo O presente trabalho analisou o perfil das publicações relacionadas à saúde do idoso no periódico Ciência \& Saúde Coletiva, em termos de sua evolução quantitativa, abordagem metodológica, temática e instituições envolvidas. A seleção dos artigos considerou o título, resumo e descritores e cobriu todo o conteúdo publicado entre 1996 e 2019. Os artigos incluídos foram classificados quanto ao ano de publicação, tipo, temática, abordagem metodológica e vínculo institucional do primeiro autor. A análise dos dados baseouse em frequências absolutas e relativas, além de medidas-sintese (média) e de variabilidade (desvio-padrão). Os resultados mostraram um consistente crescimento das publicações acerca da saúde do idoso na Ciência \& Saúde Coletiva, com predominância da abordagem quantitativa, voltada majoritariamente para a investigação de temática relacionada às condições de saúde e ao uso de serviços e insumos de saúde. Na maior parte deles, $o$ autor principal era vinculado a uma instituição de ensino/pesquisa de caráter público, localizada na região Sudeste. Os resultados indicam que o periódico tem contribuído para dar uma maior visibilidade à saúde do idoso, mas que as publicações sobre esse tema reproduzem a desigualdade vista na produção científica nacional.

Palavras-chave Saúde do Idoso, Envelhecimento, Publicações científicas 


\section{Introdução}

O envelhecimento populacional consiste na mudança da estrutura etária de uma população, com o aumento da proporção de pessoas idosas na sua composição, em decorrência da conjugação da queda da fecundidade e da queda da mortalidade, concentrada nas idades mais avançadas. No Brasil, esse processo acelerou-se na última década do século XX. Em cinco décadas, o número médio de filhos por mulher diminuiu de 6,3 (1960) para 1,9 (2010), situando-se ligeiramente abaixo do nível de reposição ${ }^{1}$. Os idosos $(60$ anos ou mais), que em 1950 representavam $4,9 \%$ da população brasileira, somavam $7,9 \%$ em 2000 , $10,2 \%$ em 2010, e estima-se que essa proporção alcançará $23,9 \%$ em $2040^{2}$.

No nível individual, o maior número de anos vividos tende a impactar negativamente a saúde, pelos danos celulares e diminuição das reservas fisiológicas que acometem um organismo envelhecido. Nesse estágio de vida, a ocorrência de condições crônicas de saúde (como por exemplo, hipertensão e diabetes) e o declínio das capacidades cognitiva e física prejudicam a autonomia do idoso e comprometem sua qualidade de vida ${ }^{3}$. No nível coletivo, o envelhecimento populacional impõe desafios à sociedade, no sentido de promover o envelhecimento saudável, de modo a evitar que o prolongamento da existência se dê com o predomínio da precarização da saúde e da perda de qualidade de vida. Os desafios colocados vão além da atenção a um perfil de morbidade populacional, em que o predomínio de condições crônicas de saúde exige dos serviços de saúde uma atenção continuada. Assim, questões tão distintas (porém, interligadas) como os ambientes físico, socioeconômico e cultural que favoreçam o envelhecimento saudável, a violência, a construção de redes de proteção social, o cuidado domiciliar e de longa duração e os benefícios sociais decorrentes do investimento no envelhecimento saudável passam a integrar o rol de desafios e ampliam o escopo das reflexões acerca da questão $0^{3,4}$.

Historicamente, a ciência sempre é chamada a dar respostas aos desafios colocados pelas sociedades em transformação, e nesse aspecto, as respostas devem caminhar em consonância com os interesses da sociedade ${ }^{5}$. Se o envelhecimento populacional nos aponta inicialmente a demografia como a ciência que naturalmente se encarregaria da sua investigação, suas repercussões na saúde (individual e coletiva) aproximam-no de um espectro científico mais abrangente, que extrapola as especificidades da ciência demográfica. Nesse contexto, a saúde coletiva constitui um campo multidisciplinar de saberes (conhecimentos teóricos explicativos ou interpretativos) e práticas (intervenções) voltadas para a prevenção, o controle e erradicação dos problemas de saúde, em nível populacional. No campo da saúde coletiva, os conhecimentos oriundos de diferentes disciplinas, abrigadas nas ciências da saúde e nas ciências humanas e sociais, são empregados no desenvolvimento de estudos cooperativos interdisciplinares, que buscam produzir conhecimento acerca da saúde de grupos populacionais específicos ${ }^{6}$.

Em 1996, logo após a X Conferência Nacional de Saúde, a Associação Brasileira de Saúde Coletiva (Abrasco) lançou o periódico Ciência \& Saúde Coletiva (C\&SC), com o propósito de constituir um espaço para divulgação e reflexão sobre os conhecimentos (teóricos e práticos) das disciplinas científicas que compõem o campo da Saúde Coletiva. O periódico se propunha a integrar a multiplicidade de olhares científicos que conformam esse campo de saber e prática, constituindo um veículo de comunicação dos avanços do conhecimento em saúde pública, com o fito de subsidiar as políticas públicas, a gestão e ofertas de serviços no âmbito do Sistema Único de Saúde (SUS) ${ }^{5}$.

Considerando as repercussões do envelhecimento populacional para a saúde do idoso (especialmente na perspectiva coletiva) e a missão e o propósito assumidos pela C\&SC, o presente trabalho pretendeu descrever e analisar o perfil das publicações relacionadas à saúde do idoso nesse periódico, em termos de sua evolução quantitativa, abordagem metodológica, temática contemplada e instituições envolvidas.

\section{Metodologia}

Para triagem das publicações acerca da saúde do idoso na Ciência \& Saúde Coletiva (C\&SC), pesquisou-se no site do periódico todo o conteúdo publicado entre 1996 e 2019. A seleção incluiu artigos originais, teóricos (ensaios ou artigo de debate) e de revisão da literatura; foram excluídos os comentários (relativos aos artigos de debate), as cartas ao editor e as resenhas. A seleção dos artigos foi baseada no título e resumo. Quando a leitura do resumo não permitiu uma tomada de decisão quanto à inclusão (ou não) do artigo, este foi lido na íntegra. Esse levantamento foi realizado por dois pesquisadores, individual- 
mente, e posteriormente, o material selecionado foi cotejado para identificação e resolução de discrepâncias e inconsistências.

Os artigos incluídos foram classificados quanto ao ano de publicação, tipo de artigo, temática, abordagem metodológica e instituição do primeiro autor. Em relação ao tipo, os artigos foram classificados em original, de revisão e teórico (opinião ou ensaio). A leitura do objetivo permitiu identificar o objeto de estudo, que foi utilizado na classificação do artigo quanto ao tema. As categorias utilizadas na classificação temática foram definidas consensualmente pelos autores, e posteriormente agregadas em categorias mais abrangentes. A classificação relativa à abordagem metodológica foi restrita aos artigos empíricos, classificados em quantitativos, qualitativos ou mistos (utilização simultânea das abordagens quantitativa e qualitativa). $\mathrm{O}$ vínculo institucional do primeiro autor foi utilizado na definição da instituição responsável pelo artigo, em termos de sua localização, tipo e identificação (nome). A localização da instituição foi baseada nas cinco grandes regiões geográficas brasileiras; o tipo de instituição considerou as categorias ensino/pesquisa, ensino somente e outros órgãos públicos (que não os de ensino e pesquisa). Além disso, na tipificação das instituições, classificouse quanto à gestão, se pública ou privada.

A análise dos dados baseou-se em frequências absolutas e relativas, além de medidas-síntese (média) e de variabilidade (desvio-padrão). Para análise da evolução quantitativa no tempo, os anos-calendário foram agrupados em intervalos de cinco anos (quinquênios), com exceção do período mais recente, entre 2016 e 2019, que constituiu um quadriênio. Para cada período de anos, calculou-se o número total e a média anual de artigos publicados, além do que representaram, em termos proporcionais, em relação ao total dos artigos publicados pela revista. Os resultados foram apresentados em tabelas e gráficos, sendo que para as variáveis tema e nome da instituição, foram apresentadas em tabela apenas as $10 \mathrm{ca}-$ tegorias mais frequentes, sendo as demais agregadas em uma categoria denominada "outras". Os dados foram armazenados e analisados com recurso do programa Excel $^{\circledR}$, do pacote Microsoft Office, versão 2010.

\section{Resultados}

Entre 1996 (ano de lançamento) e 2019, a Revista Ciência \& Saúde Coletiva publicou 396 artigos que contemplaram a saúde da pessoa idosa, com uma média 17,2 (dp = 19,1) por ano (o primeiro deles foi publicado em 2002). O Gráfico 1 descreve a evolução do quantitativo de artigos, em termos absolutos e relativos (ao total de publicações do periódico) entre os anos de 2001 e 2019. Em ambos os casos, observa-se um crescimento consistente: foram 11 artigos publicados no período 2001-2005 (que representaram 3,7\% do total publicado) e 148 (9,6\% do total) publicados no período 2016-2019; a década iniciada em 2011 concentrou $77,8 \%(\mathrm{n}=308)$ das publicações sobre saúde do idoso. Em termos médios, a tendência de crescimento foi semelhante: 2,2 artigos/ ano no período 2001-2005, 15,4 entre 2006-2010, 32,0 entre 2011-2016 e 37,0 no período de anos mais recente (2016-2019) (dados não mostrados no gráfico).

Quanto ao tipo de artigo, 85,4\% ( $\mathrm{n}=338)$ são empíricos, sendo os restantes (14,6\%) de revisão (bibliográfica ou documental) e teóricos. Entre os artigos empíricos, 79,3\% utilizaram metodologia quantitativa, $16,0 \%$ a qualitativa e $4,7 \%$ associaram as duas metodologias. Os objetos de investigação foram variados, com o predomínio daqueles que foram abrigados na categoria Condições de Saúde, e que somaram 136 (ou 34,3\%), seguindo-se Serviços e Insumos de Saúde ( $\mathrm{n}=48$ ou $12,1 \%)$, Violência/Suicídio $(\mathrm{n}=42$ ou 10,6\%) e Capacidade Funcional ( $\mathrm{n}=26$ ou 6,6\%). Uma descrição mais detalhada dessas informações pode ser vista na Tabela 1 .

Do total de artigos publicados, 377 (95,2\%) eram brasileiros, ou seja, o primeiro autor tinha vínculo com uma instituição de pesquisa/ensino brasileira. As publicações de origem internacional são da Colômbia, Cuba, Espanha, Estados Unidos, México, Portugal, Suíça, Turquia e Uruguai, sendo 19 (4,8\%) do total de publicações em saúde do idoso. Entre os artigos publicados em outra língua, 143 foram publicados em inglês e cinco em espanhol. Considerando a distribuição das instituições de afiliação por região geográfica, três quartos das publicações são das regiões Sudeste e Sul (56,8\% e 19,6\%, respectivamente), seguindo-se a Nordeste $(15,1 \%)$, Centro-Oeste $(6,9 \%)$ e Norte $(1,6 \%)$. A quase totalidade dos artigos ( $\mathrm{n}=342$ ou $96,6 \%$ ) têm o autor principal vinculado a uma instituição de ensino e/ou pesquisa; os demais são de serviços públicos (órgãos federais, secretarias municipais ou estaduais de saúde). Das instituições às quais o primeiro autor do artigo é vinculado, $85,8 \%(\mathrm{n}=326)$ são de caráter público (federal ou estadual). Entre estas, destacam-se a Fundação Oswaldo Cruz (Fiocruz) 


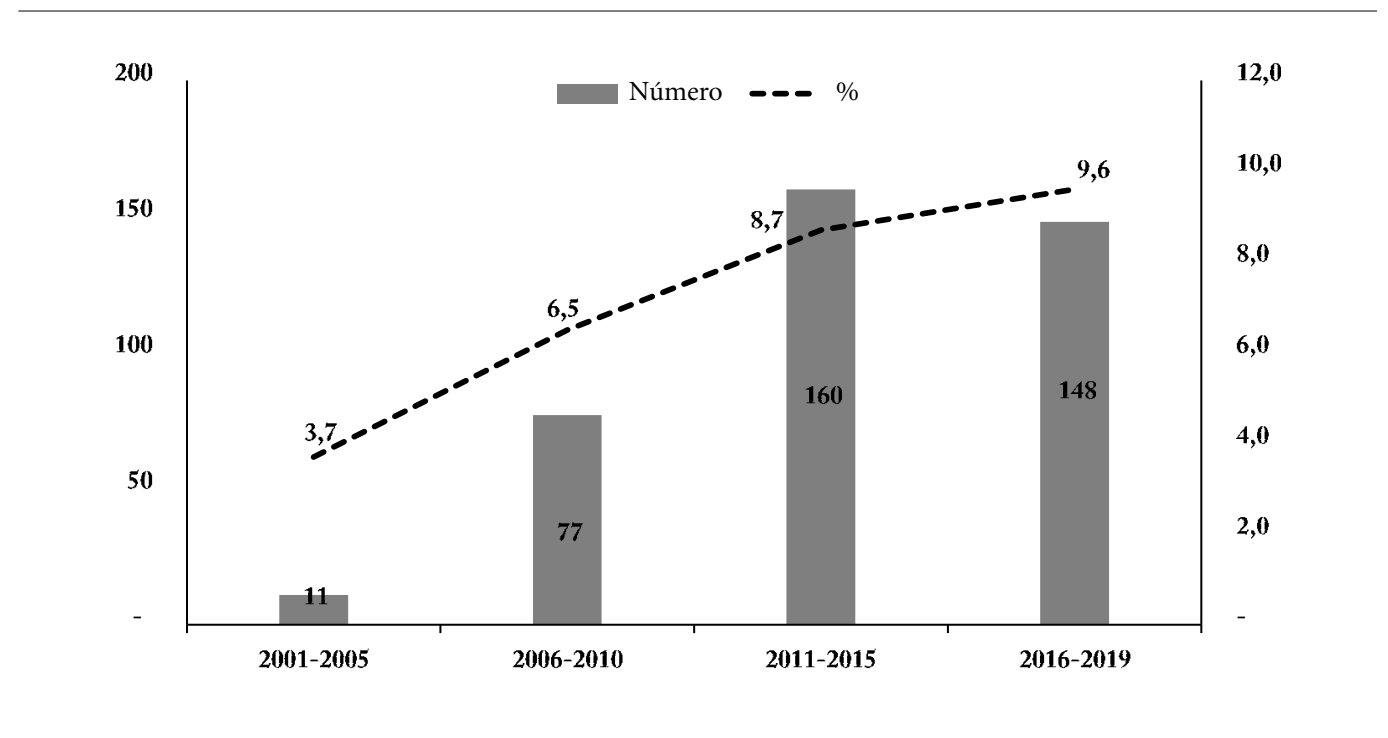

Gráfico 1. Evolução do quantitativo de artigos publicados na Revista Ciência \& Saúde Coletiva (número total e percentual em relação ao total geral de artigos), 1996 a 2019.

$(\mathrm{n}=50)$ e a Universidade Estadual de Campina (UNICAMP) $(\mathrm{n}=30)$, que juntas, respondem por aproximadamente um quarto $(24,3 \%)$ das publicações. A Tabela 2 apresenta uma descrição detalhada desses resultados, destacando as dez instituições de pesquisa de caráter público com maior número de publicações.

\section{Discussão}

O presente estudo mostrou um consistente crescimento das publicações acerca da saúde do idoso na Ciência \& Saúde Coletiva, em termos absolutos (número de artigos publicados), e relativos (proporção do total de artigos publicados no periódico). Nos artigos, predominou a abordagem quantitativa, voltada majoritariamente para a investigação de temática relacionada às condições de saúde e ao uso de serviços e insumos de saúde. Além disso, na maior parte deles, o primeiro autor era vinculado a uma instituição de ensino/ pesquisa de caráter público, localizada na região Sudeste.

A tendência de crescimento do número de publicações sobre a saúde do idoso acentuou-se nesta década, praticamente quadruplicando nos anos 2011-2019, se comparado à década anterior (2002-2010). Esse crescimento se dá concomitantemente ao aumento do volume de artigos publicados pelo periódico na mesma década (3.386 publicados entre 2011-2019 contra 1.444 publicados entre 2001-2010), porém, numa velocidade maior. O resultado foi uma maior participação dessa área do conhecimento no conjunto de publicações do periódico (ao final da década, praticamente um em cada 10 artigos abordaram a saúde do idoso).

Observa-se uma carência de artigos científicos com objetivos semelhantes ao nosso nos periódicos nacionais, na área de saúde coletiva. Identificamos dois estudos de revisão, ambos de cunho bibliométrico, que abordaram a evolução da produção científica sobre a saúde do idoso. No primeiro deles ${ }^{7}$ os autores investigaram a força de trabalho científica (grupos de pesquisa) que se dedicava ao tema, tendo diagnosticado uma reduzida capacidade de reprodução dessa força de trabalho, em função da heterogeneidade dos grupos de pesquisa e da baixa inserção em programas de pós-graduação. À época da publicação, anteviam um percurso longo para a consolidação científica dos grupos de pesquisa voltados especificamente para o estudo do envelhecimento populacional. O segundo ${ }^{8}$, de publicação mais recente, argumentou que o surgimento e consolidação de um periódico científico voltado para os estudos na área de geriatria e gerontologia era uma evidência do crescimento da produção científica sobre a saúde do idoso. 
Tabela 1. Distribuição das publicações da Revista Ciência \& Saúde Coletiva, segundo metodologia utilizada e temática central, 2002-2019.

\begin{tabular}{|c|c|c|}
\hline Variáveis & $\mathbf{N}$ & $\%$ \\
\hline \multicolumn{3}{|l|}{ Metodologia utilizada $(\mathrm{n}=338)^{\mathrm{a}}$} \\
\hline Quantitativa & 268 & 79,3 \\
\hline Qualitativa & 54 & 16,0 \\
\hline Qualitativa e Quantitativa & 16 & 4,7 \\
\hline \multicolumn{3}{|l|}{ Tema principal $(\mathrm{n}=396)$} \\
\hline Condições de saúde & 136 & 34,3 \\
\hline Serviços e Insumos de Saúde & 48 & 12,1 \\
\hline Violência/Suicídio & 42 & 10,6 \\
\hline Capacidade Funcional & 26 & 6,6 \\
\hline Qualidade de Vida & 23 & 5,8 \\
\hline Cuidado ao Idoso & 20 & 5,1 \\
\hline Fragilidade & 13 & 3,3 \\
\hline Aspectos Socioeconômicos & 9 & 2,3 \\
\hline Gênero & 9 & 2,3 \\
\hline Promoção/Educação em saúde & 7 & 1,8 \\
\hline Outros $^{\mathrm{b}}$ & 63 & 15,9 \\
\hline \multicolumn{3}{|c|}{ 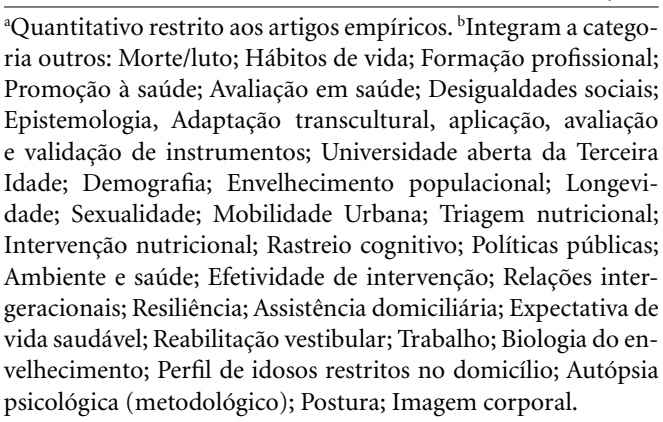 } \\
\hline
\end{tabular}

O crescimento das pesquisas sobre saúde do idoso parece refletir a mesma tendência da produção científica brasileira na área de saúde pública neste século, alimentado por uma política governamental de financiamento da ciência e tecnologia, iniciada em $2000^{9}$, a partir de uma agenda de prioridades definidas pelo Ministério da Saúde em um processo amplo de consulta ${ }^{10}$. Certamente contribuiu para esse crescimento a implementação da Política Nacional do Idoso e da Política Nacional de Saúde do idoso, nos anos 1990, que focam o envelhecimento ativo ${ }^{11}$. Por fim, cabe mencionar que o incremento do desenvolvimento científico e tecnológico no campo da saúde é uma das competências do SUS, e isso está inscrito na carta magna nacional (artigo 200, inciso V). No entanto, as transformações políticas nos anos recentes impuseram importantes restrições ao financiamento da Educação e da Saúde, e
Tabela 2. Distribuição das publicações da Revista Ciência \& Saúde Coletiva, segundo afiliação institucional do primeiro autor, 2002-2019.

\begin{tabular}{|c|c|c|}
\hline Variáveis & $\mathbf{N}$ & $\%$ \\
\hline \multicolumn{3}{|l|}{ Localização da instituição $(\mathrm{n}=377)^{\mathrm{a}}$} \\
\hline Sudeste & 214 & 56,8 \\
\hline Sul & 74 & 19,6 \\
\hline Nordeste & 57 & 15,1 \\
\hline Centro-Oeste & 26 & 6,9 \\
\hline Norte & 6 & 1,6 \\
\hline \multicolumn{3}{|l|}{ Tipo de instituição (n=377) } \\
\hline Ensino e Pesquisa (Pública) & 326 & 82,3 \\
\hline Ensino (Privada) & 54 & 13,6 \\
\hline Órgão Público & 16 & 4,0 \\
\hline \multicolumn{3}{|l|}{$\begin{array}{l}\text { Instituição Pública de Ensino e } \\
\text { Pesquisa }(n=326)\end{array}$} \\
\hline Fundação Oswaldo Cruz (Fiocruz) & 50 & 15,3 \\
\hline $\begin{array}{l}\text { Univ. Est. de Campinas } \\
\text { (UNICAMP) }\end{array}$ & 30 & 9,2 \\
\hline Universidade de São Paulo (USP) & 17 & 5,2 \\
\hline Univ. Fed. Minas Gerais (UFMG) & 16 & 4,9 \\
\hline Univ. Est. Rio de Janeiro (UERJ) & 15 & 4,6 \\
\hline Univ. Fed. Santa Catarina (UFSC) & 13 & 4,0 \\
\hline $\begin{array}{l}\text { Univ. Est. de Montes Claros } \\
\text { (UNIMONTES) }\end{array}$ & 13 & 4,0 \\
\hline Univ. Fed. Pelotas (UFPel) & 12 & 3,7 \\
\hline $\begin{array}{l}\text { Univ. Fed. Triângulo Mineiro } \\
\text { (UFTM) }\end{array}$ & 11 & 3,4 \\
\hline Univ. Fed. Pernambuco (UFPE) & 10 & 3,1 \\
\hline Outras & 139 & 42,6 \\
\hline
\end{tabular}

no caso dessa situação não ser revertida, não será surpresa um arrefecimento do crescimento da produção científica da saúde coletiva (incluído saúde do idoso) nos próximos anos.

A abordagem quantitativa esteve presente em mais de $80 \%$ dos estudos relacionados à saúde do idoso publicados na revista. Esse predomínio é consistente com o observado em diferentes linhas de investigação no campo da saúde coletiva, que demonstra o predomínio dos estudos epidemiológicos ${ }^{8,11-13}$. A Epidemiologia é uma disciplina científica de base quantitativa, e em sua definição, assume o compromisso com a promoção da saúde de populações humanas, produzindo conhecimento sobre o comportamento de eventos de saúde e seus determinantes em nível populacional $^{14}$. Historicamente, a Epidemiologia tem sido um dos pilares da saúde coletiva, formulando diagnósticos de saúde e reconhecendo mudanças 
no estado de saúde das populações ao longo do tempo, identificando causas, fatores de risco e determinantes de problemas de saúde, avaliando o risco individual e coletivo para o adoecimento e constatando as necessidades dos serviços de saúde. Ao estudar aspectos sociais da doença, ela ajuda a fornecer um quadro completo das doenças e agravos à saúde, respondendo a questões que a clínica isoladamente nem sempre consegue ${ }^{15}$.

Assim, o conhecimento produzido por ela torna-se de fundamental importância para a atuação dos serviços de saúde e sua avaliação. No Brasil, os estudos epidemiológicos e a saúde coletiva ganharam impulso nos anos 1970, quando foram criados o primeiro curso de pós-graduação em saúde coletiva (início da década) e a Associação Brasileira de Saúde Coletiva (Abrasco) (no final da década). O compromisso da comunidade de epidemiologistas brasileiros com a saúde da população e com o desenvolvimento e aprimoramento do SUS é responsável pela consolidação da pesquisa epidemiológica no país, e pela pujança da sua produção científica na última década, que cresceu a uma velocidade tão rápida quanto a de outros campos científicos na área de ciências da saúde ${ }^{16}$. Esse importante crescimento está, portanto, representado na produção sobre saúde do idoso da Ciência \& Saúde Coletiva, como descrito nos resultados do presente artigo.

A presença minoritária dos estudos qualitativos pode ser consequente ao fato de que o reconhecimento de sua cientificidade é recente: esses estudos eram considerados relatos regidos pela subjetividade ou, no máximo, estudos heurísticos ou pré-científicos ${ }^{17}$. Nas investigações qualitativas, o objeto de estudo não é o evento de saúde em si, mas o seu significado individual e coletivo, e o objetivo do pesquisador não é explicar o comportamento do evento de saúde, mas sim interpretar e compreender o que ele significa para quem o experimenta. Isso diferencia a abordagem qualitativa da quantitativa, porque a interpretação do evento pelo cientista se dá na perspectiva do entrevistado, e não dos pressupostos teóricos que o primeiro carrega. Para a saúde coletiva, a pesquisa qualitativa ganha em importância porque é em torno do significado do evento e do seu compartilhamento cultural que os indivíduos e os grupos sociais organizam a sua vida ${ }^{16}$. Portanto, as ações implementadas pelos indivíduos frente ao problema de saúde decorrerão da maneira como os percebem e os valorizam, e precisam ser considerados pelos serviços de saúde no atendimento das necessidades de saúde da população.
Nessa perspectiva, vários eventos de saúde têm merecido uma investigação sob olhar qualitativo, como é o caso da autoavaliação da saúde ${ }^{18}$, capacidade funcional ${ }^{19}$, uso de medicamentos ${ }^{20,21}$, violência contra o idoso ${ }^{22}$ e relação profissional de saúde-paciente ${ }^{23}$ para mencionar alguns. São estudos que exemplificam as possibilidades do método qualitativo na abordagem de eventos cuja complexidade interna limita o alcance das abordagens metodológicas sustentadas na objetividade e quantificação, possibilitando conhecê -los em maior profundidade. O significado dado aos fenômenos naturais (entre os quais incluemse os eventos de saúde) e às situações reais define as suas consequências, motivando assim as ações humanas. A pesquisa qualitativa trabalha com a subjetividade, a intencionalidade e o significado, a partir da interação com o concreto e o morfológico. Desse modo, nas investigações no campo da saúde coletiva, os métodos quantitativo e qualitativo não devem ser vistos como oposição, mas como abordagens complementares ${ }^{24}$.

A diversidade temática observada nas publicações reflete a complexidade do conceito de saúde e quão multifacetado ele é, especialmente em relação ao idoso. Entre os temas abordados, os problemas de saúde, a utilização de serviços e insumos de saúde e a violência foram os mais frequentes, indicando que os desdobramentos negativos à saúde do idoso (doenças e agravos, presentes isolada ou concomitantemente) e a demanda que representam para os serviços de saúde foram as questões que mais têm instigado os pesquisadores. A título de exemplo, em 2000, as doenças crônicas não transmissíveis constituíam a linha de pesquisa mais frequente entre os grupos de pesquisa brasileiros cujas investigações se relacionavam, de alguma forma, com o envelhecimento humano ${ }^{7}$.

Na categoria Condições de Saúde, chama a atenção o espaço reservado à saúde bucal. A publicação de artigos indexados voltados para a saúde bucal do idoso (e da população geral) tem aumentado, especialmente a partir de 2006. Esse incremento tem se caracterizado por uma descentralização dos periódicos tradicionais da saúde coletiva, indicando uma renovação temática e de abordagens metodológicas, com destaque para a C\&SC no início desta década (2011) ${ }^{25}$. A saúde oral é um componente da saúde geral, com reflexos sobre o bem-estar físico, psicológico e social do indivíduo. A necessidade de pesquisas adicionais, multidisciplinares, no domínio da odontologia geriátrica ainda é percebida ${ }^{26}$. Na América Latina, por exemplo, são poucos os estudos sobre 
a situação de saúde bucal dos idosos, e eles evidenciam que ela é uma característica compartilhada pelos idosos da região. O planejamento de políticas públicas para a saúde oral é prejudicado pela carência de informações epidemiológicas, e faz-se necessário a inclusão da odontologia geriátrica nos currículos das escolas de odontologia ${ }^{27}$.

No tocante à categoria Uso de Serviços/Insumos de Saúde, observou-se um predomínio dos estudos voltados para a investigação do uso de medicamentos. Não seria um exagero afirmar que o medicamento é um insumo onipresente no cenário de atenção à saúde, dada a sua efetividade no manejo dos mais diversos problemas de saúde. A comorbidade é comum entre idosos ${ }^{28}$, o que torna o idoso mais propenso ao uso de medicamentos. Entre idosos, a polifarmácia (uso simultâneo de medicamentos) ${ }^{29}$ é frequente ${ }^{30,31}$, e com ela, aumenta a probabilidade de ocorrência de reações medicamentosas adversas e o uso de medicamentos potencialmente inadequados $(\mathrm{MPI})^{32}$. Em um contexto em que o uso do medicamento (frequentemente necessário) pode acarretar tanto ou mais riscos ${ }^{33,34}$ que benefícios, é natural que a atenção dos pesquisadores esteja voltada para o entendimento do fenômeno e suas implicações para a saúde coletiva, sobretudo entre idosos. Identificamos um único estudo ${ }^{35}$ que buscou sistematizar as publicações sobre uso de medicamentos no Brasil e América Latina. Essa revisão, que analisou os estudos publicados até 2004 e restrita às bases Scielo e Lilacs, detectou um predomínio de estudos quantitativos, com o foco na prevalência e perfil do uso de medicamentos, e uma carência de estudos desenvolvidos junto à população idosa. São poucos também os estudos longitudinais voltados para esse tema, que podem contribuir de maneira importante para a detecção de situações de risco no uso de medicamentos pelos idosos ${ }^{33,36,37}$.

A violência é um fenômeno multifacetado, definido por atos perpetrados individual ou institucionalmente com a intenção de causar prejuízo físico, moral, sexual ou psicológico a outrem. Uma revisão da literatura ${ }^{4}$ publicada ao final da década passada mostrou que a produção científica brasileira sobre a violência contra o idoso havia aumentado nos primeiros dez anos deste século, se comparada ao observado na década anterior. As quedas (suas circunstâncias e consequências) e as causas externas e a violência em geral (investigados na perspectiva epidemiológica) foram os tipos de violência mais abordados nos estudos identificados. Apesar do crescimento constatado, os autores alertam para a pouca atenção dada a alguns aspectos da violência contra o idoso, como a sua prevenção, a percepção do fenômeno entre cuidadores e profissionais de saúde e a violência doméstica, que necessitam ser mais frequentemente investigados. A carência de estudos sobre a violência sexual contra idosos e suas intersecções com a violência doméstica é percebida também na literatura internacional. Embora os estudos existentes identifiquem a mulher (com incapacidade física e cognitiva, especialmente) como a vítima mais frequente, poucos estudos têm abordado os impactos (físicos e psicológicos) da violência, as características do agressor, as necessidades das vítimas (que são diferentes das vítimas jovens) e a experiência dos sobreviventes ${ }^{38}$. Nossos resultados indicam que a tendência de crescimento de publicações sobre a violência contra o idoso observada na década passada está presente na C\&SC.

Embora os artigos em língua portuguesa tenham predominado, mais de um terço deles $(36,1 \%)$ foram publicados em inglês (a maior parte correspondendo a publicação bilíngue), resultante do esforço de internacionalização do periódico. A maioria dos artigos publicados são vinculados a instituições de ensino/pesquisa de caráter público e localizadas na região Sudeste, com destaque para a Fiocruz, UNICAMP e USP. A predominância da região Sudeste é observada em outras linhas de investigação da saúde coletiva, como a nutrição ${ }^{12}$. No nosso estudo, entre as dez instituições que mais publicaram, apenas duas (UFPEL e UFPE) não estão localizadas na região Sudeste. As instituições destacadas abrigam grupos de pesquisas com projetos importantes com foco na saúde e bem-estar do idoso, como é o caso dos Projetos Bambuíi ${ }^{39}$ e ELSI-Brasil (da Fiocruz) ${ }^{9}$, o projeto SABE (USP) $)^{40}$ e o Projeto FIBRA (UNICAMP) ${ }^{41}$. Uma consulta à lista de grupos de pesquisa do $\mathrm{CNPq}$ na área de saúde coletiva e que tinham no nome do grupo as palavras "idoso", "envelhecimento", "melhor idade", "gerontologia/geriatria" revelou que, do total de 32 grupos registrados, 12 são da região Sudeste (cinco em São Paulo, quatro no Rio de Janeiro e três em Minas Gerais $)^{42}$. Esses achados evidenciam a desigualdade na produção científica brasileira sobre saúde do idoso, concentrados nos estados mais desenvolvidos economicamente e com maior número de instituições públicas de ensino/pesquisa. Essas assimetrias regionais, embora tenham reduzido ao longo do tempo, ainda permanecem, com supremacia da Região Sudeste. A análise do triênio 2007-2009 mostra que $54,3 \%$ de toda produção científica brasilei- 
ra estava concentrada nessa Região, que também somava $63,2 \%$ do total produzido na área da Ciências da Saúde ${ }^{43}$.

O presente estudo apresenta limites que dificultam a comparação dos seus resultados com o conteúdo publicado em outros periódicos de saúde coletiva, nacionais e internacionais. Tivemos dificuldades em identificar estudos semelhantes ao nosso, quanto ao desenho e objetivo nos principais bancos de dados bibliográficos, como BIREME e PubMed. Não constituiu objetivo deste estudo proceder a um levantamento da produção nacional e internacional nos moldes do que foi feito em relação à C\&SC, e dessa forma, o mapeamento dessa produção baseouse em artigos de revisão que contemplaram, em algum aspecto, objetos relacionados à saúde do idoso. Além disso, a precisão de alguns resultados específicos ficou prejudicada, como é o caso da vinculação institucional da pesquisa. A opção por considerar, no estudo, apenas o primeiro autor na avaliação da produção institucional, certamente subestima a participação de outras instituições às quais os coautores dos artigos estão vinculados. Por outro lado, a consulta a todos os números publicados pela revista e a cuidadosa categorização das informações contidas nesses trabalhos contribui para a reflexão da produção sobre saúde do idoso nesse periódico, estando em consonância, possivelmente, com o crescimento dessa área de conhecimento no país e no mundo.

\section{Considerações finais}

O crescimento (absoluto e relativo) do espaço ocupado pela produção científica acerca da saúde do idoso na C\&SC é uma boa notícia, pois aponta para uma maior visibilidade dada a essa questão no campo da saúde coletiva. São constatações positivas também o espaço reservado à metodologia qualitativa (ainda que minoritário) e à pluralidade temática. A abordagem qualitativa é de grande importância para alcançar uma visão mais aprofundada das questões afeitas à saúde do idoso, e a diversidade temática reflete o quão complexo é esse campo de investigação, revelando a dificuldade de ser abarcado sem a multidisciplinaridade.

Por outro lado, a análise das publicações mostrou que alguns temas ainda aguardam um olhar mais atento dos pesquisadores da saúde coletiva, e que a desigualdade na produção científica brasileira está presente nos estudos sobre a saúde do idoso. No primeiro caso, questões como o cuidado, as redes de segurança social e o capital social, a sexualidade, o ambiente e a religiosidade carecem de mais investigações junto à população idosa; no segundo caso, observa-se que as pesquisas sobre o envelhecimento permanecem concentradas na região Sudeste.

A ciência tem um compromisso com a sociedade, na produção de respostas às suas demandas e de soluções para os seus problemas. Assim, ela pode sustentar a adoção de políticas e práticas baseadas em evidências científicas, que resultem em ações de saúde de melhor custo-efetividade ${ }^{44}$. Mas o conhecimento produzido necessita de instrumentos de divulgação, para que possa ser apropriado, inicialmente pela comunidade científica, e ao final, pela sociedade como um todo. Nesse aspecto, este periódico tem constituído um espaço privilegiado para a divulgação do conhecimento produzido pelo campo da saúde coletiva e tem a pretensão de contribuir para a construção e consolidação do SUS. Os estudos publicados no periódico que contemplem a saúde do idoso certamente instigarão pesquisadores e gestores a aprofundar a reflexão sobre a necessidade dos serviços de saúde (especialmente o SUS), bem como o diálogo com os gestores, na busca de soluções para as demandas apresentadas por esse crescente segmento etário. 


\section{Colaboradores}

JOA Firmo, SV Peixoto, GA Souza e AI Loyola Filho, participaram da concepção, planejamento, coleta de dados, análise e interpretação dos dados; contribuíram na elaboração, revisão e aprovação da versão final do artigo.

\section{Referências}

1. Vasconcelos AMN, Gomes MM. Transição demográfica: a experiência brasileira. Epidemiol Serv Saúde 2012; 21(4):539-548.

2. United Nations (UN). Department of Economic and Social Affairs, Population Division. World Population Prospects 2019 [Internet]. New York: UN; 2020 [acessado 2020 Maio 1]. Disponível em: http://esa.un.org/ unpd/wpp/

3. Beard JR, Officer A, Carvalho IA, Sadana R, Pot AM, Michel JP, Lloyd-Sherlock P, Epping-Jordan JE, Peeters GMEE, Retno Mahanani W, Thiyagarajan JA, Chatterji S. The World report on ageing and health: a policy framework for healthy ageing. Lancet 2016; 387(10033):2145-2154.

4. Minayo MCS, Souza ER, Paula DR. Revisão sistemática da produção acadêmica brasileira sobre causas externas e violências contra a pessoa idosa. Cien Saude Colet 2010; 15(6):2719-2728.

5. Minayo MSC, Gomes R. Ciência \& Saúde Coletiva no contexto nacional e internacional da divulgação científica. Cien Saude Colet 2015; 20(7):2013-2022.

6. Luz MT. Complexidade do Campo da Saúde Coletiva: multidisciplinaridade, interdisciplinaridade, e transdisciplinaridade de saberes e práticas - análise sócio -histórica de uma trajetória paradigmática. Saúde Soc 2009; 18(2):304-311.

7. Prado SD, Sayd JD. A pesquisa sobre envelhecimento humano no Brasil: pesquisadores, temas e tendências. Cien Saude Colet 2004; 9(3):763-772.

8. Jerez-Roig J, Guedes MBOG, Silva JMD, Lima KC. Análise da produção científica da Revista Brasileira de Geriatria e Gerontologia: uma revisão bibliométrica. Rev Bras Geriatr Gerontol 2014; 17(3):659-671.

9. Victora CG, Barreto ML, Leal MC, Monteiro CA, Schmidt MI, Paim JS, Bastos FIPM, Almeida CM, Bahia L, Travassos CMR, Reichenheim M, Barros FC. Health conditions and health-policy innovations in Brazil: the way forward. Lancet 2011; 377(9782):2042-2053.

10. Akerman M, Fischer A. Agenda Nacional de Prioridades na Pesquisa em Saúde no Brasil (ANPPS): foco na subagenda 18 - Promoção da Saúde. Saúde Soc 2014; 23(1):180-190.

11. Barros MBA, Golbaum M. Desafios do envelhecimento em contexto de desigualdade social. Editorial. Rev Saúde Pública 2018; 52(Supl. 2):1s.

12. Canella DS, Silva ACF, Jaime PC. Produção científica sobre nutrição no âmbito da Atenção Primária à Saúde no Brasil: uma revisão de literatura. Cien Saude Colet 2013; 18(2):297-308.

13. Pagotto V, Bachion MM, Silveira EA. Autoavaliação da saúde por idosos brasileiros: revisão sistemática da literatura. Rev Panam Salud Publica 2013; 33(4):302310.

14. Last JM. A dictionary of Epidemiology. New York: Oxford University Press; 1995.

15. Morris JN. Uses of Epidemiology. BMJ 1955; 13:395401.

16. Barreto ML, Barata RB. Public health and epidemiology journals published in Brazil and other Portuguese speaking countries. Emerging Themes Epidemiol $2008,5: 18$ 
17. Turato ER. Métodos qualitativos e quantitativos na área da saúde: definições, diferenças e seus objetos de pesquisa. Rev Saúde Pública 2005; 39(3):507-514.

18. Fonseca MGUP, Firmo JOA, Loyola Filho AI, Uchoa E. Papel da autonomia na autoavaliação da saúde do idoso. Rev Saúde Pública 2010; 44(1):159-165.

19. Pereira JK, Giacomin KC, Firmo JOA. A funcionalidade e incapacidade na velhice: ficar ou não ficar quieto. Cad Saúde Pública 2015; 31(7):1451-1459.

20. Alvarenga JM, Loyola Filho AI, Giacomin KC, Uchoa E, Firmo JOA. Uso de benzodiazepínicos entre idosos: o alívio de “jogar água no fogo”, não pensar e dormir. Rev Bras Geriatr Gerontol 2015; 18(2):249-258.

21. Loyola Filho AI, Lima-Costa MF, Uchoa E. Bambuí Project: a qualitative approach to self-medication. Cad Saúde Pública 2004; 20(6):1661-1669.

22. Silva MJ, Oliveira TM, Joventino ES, Moraes GLA. A violência na vida cotidiana do idoso: um olhar de quem a vivência. Rev Eletrônica Enferm 2008; 10(1):124-136.

23. Thomas H, Best M, Mitchell G. Whole-person care in general practice: the doctor-patient relationship. Australian J General Practice 2020; 49(3):139-144.

24. Minayo MCS, Sanches O. Quantitativo-Qualitativo: Oposição ou Complementaridade? Cad Saúde Pública 1993; 9(3):239-262.

25. Celeste RK, Warmling CM. Produção bibliográfica brasileira da Saúde Bucal Coletiva em periódicos da saúde coletiva e da odontologia. Cien Saude Colet 2014; 19(6):1921-1932.

26. Ástvaldsdóttir Á, Boström A-M, Davidson T, Gabre P, Gahnberg L, Englund GS, Skott P, Ståhlnacke K, Tranaeus S, Wilhelmsson H, Wårdh I, Östlund P, Nilsson M. Oral health and dental care of older persons - A systematic map of systematic reviews. Gerodontology 2018; 35(4):290-304.

27. León S, De Marchi RJ, Tôrres LH, Hugo FN, Espinoza I, Giacaman RA. Oral health of the Latin American elders: What we know and what we should do - Position paper of the Latin American Oral Geriatric Group of the International Association for Dental Research. Gerodontology 2018; 35(2):71-77.

28. Theme Filha MM, Souza Junior PRB, Damacena GN, Szwarcwald CL. Prevalência de doenças crônicas não transmissíveis e associação com autoavaliação de saúde: Pesquisa Nacional de Saúde, 2013. Rev Bras Epidemiol 2015; 18 (Supl. 2):83-96.

29. Rollason V, Vogt N. Reduction of Polypharmacy in the Elderly A Systematic Review of the Role of the Pharmacist. Drugs Aging 2003; 20(11):817-832.

30. Payne RA. The epidemiology of polypharmacy. Clinical Med 2016; 16(5):465-469.

31. Loyola Filho AI, Firmo JOA, Uchoa E, Lima-Costa MF. Birth cohort differences in the use of medications in a Brazilian population of older elderly: the Bambuí Cohort Study of Aging (1997 and 2008). Cad Saúde Pública 2011; 27(Supl. 3):S435-S443.

32. The American Geriatrics Society. American Geriatrics Society 2019 Updated AGS Beers Criteria ${ }^{\circledR}$ for Potentially Inappropriate Medication Use in Older Adults. J Am Geriatr Soc 2019; 67(4):674-694.
33. Nascimento MMG, Mambrini JVM, Lima-Costa MF, Firmo JOA, Peixoto SV, Loyola Filho AI. Potentially inappropriate medications: predictor for mortality in a cohort of community-dwelling older adults. Eur J Clin Pharmacol 2017; 73:615-621.

34. Park H, Satoh H, Miki A, Urushihara H, Sawada Y. Medications associated with falls in older people: systematic review of publications from a recent 5-year period. Eur J Clin Pharmacol 2015; 71:1429-1440.

35. Leite SN, Vieira M, Veber AP. Estudos de utilização de medicamentos: uma síntese de artigos publicados no Brasil e Amérca Latina. Cien Saude Colet 2008; 13(Supl.):793-802.

36. Falci DM, Mambrini JVM, Castro-Costa E, Firmo JOA, Lima-Costa MF, Loyola Filho AI. Uso de psicofármacos prediz incapacidade funcional entre idosos. Rev Saude Publica 2019; 53:21.

37. Romano-Lieber NS, Coronall Liete LP, Marques FG, Secoli SR. Sobrevida de idosos e exposição à polifarmácia no município de São Paulo: Estudo SABE. Rev Bras Epidemiol 2018; 21(Supl. 2):E180006.

38. Bows H. Sexual Violence Against Older People: A Review of the Empirical Literature. Trauma Violence Abuse 2018; 19(5):567-583.

39. Lima-Costa MF, Firmo JOA, Uchoa E. Cohort Profile: The Bambuí (Brazil) Cohort Study of Ageing. Int J Epidemiol 2011; 40:862-867.

40. Lebrão ML, Laurenti R. Saúde, bem-estar e envelhecimento: o estudo SABE no Município de São Paulo. Rev Bras Epidemiol 2005; 8(2):127-141.

41. Neri AL, Yassuda MS, Araújo LF, Eulálio MC, Cabral BE, Siqueira MEC, Santos GA, Moura JGA. Metodologia e perfil sociodemográfico, cognitivo e de fragilidade de idosos comunitários de sete cidades brasileiras: Estudo FIBRA. Cad Saúde Pública 2013; 29(4):778-792.

42. Conselho Nacional de Desenvolvimento Científico e Tecnológico (CNPq). Diretório de grupos de pesquisa [Internet]. [acessado 2020 Mar 29]. Disponível em: http/dgp.cnpq.be/dgp/faces/consulta/consulta_parametrizada.jsf

43. Sidone OJG, Haddad EA, Mena-Chalco JP. A ciência nas regiões brasileiras: evolução da produção e das redes de colaboração científica. Transinformação 2016 28(1);15-31.

44. Santos LMP, Souza LEPF, Serruya SJ, Guimarães RFN. O papel da pesquisa na consolidação do Sistema Único de Saúde (SUS) (Editorial). Cad Saúde Pública 2010; 26(9):1666-1667.

Artigo apresentado em 26/05/2020

Aprovado em 26/05/2020

Versão final apresentada em 28/05/2020 